\title{
SOME CONDITIONS FOR MANIFOLDS TO BE LOCALLY FLAT $\left.{ }^{1}\right)$
}

\author{
BY \\ C. LACHER $\left({ }^{2}\right)$
}

Consider manifolds $M \subset N$ and a subset $X$ of $M$, and assume that $M-X$ and $X$ are locally nice in $N$. The general question considered in this paper is "What conditions on $X$ imply that $M$ is nice in $N$ ?" Without mentioning the case when $N$ is three dimensional, this question has been considered before by CantrellEdwards in [9], by Cantrell in [6] and [7], by Edwards in [11], by Bryant in [5], by Lacher in [14], and elsewhere. However, in each of the above references the author restricts himself by either assuming that $M$ lies in the trivial range or by assuming that $X$ is a single point. The conditions derived in this paper make no dimensional restriction on $M$ and assume only that $X \times[0,1]$ lies in the trivial range.

The first three sections are devoted to studying embeddings of polyhedra into a manifold (in the trivial range). The polyhedra are allowed to intersect the boundary of the manifold. The results on embeddings in the trivial range constitute a major step in the proof of the main result of this paper (Theorem 4.2). The fourth and fifth sections derive some conditions for $M$ to be nice in $N$ when $X$ lies in the boundary of $M$. The last section extends these results to the case when $X$ lies in the interior of $M$, modulo a certain conjecture.

0. Definitions and notations. $R^{n}$ is euclidean $n$-space, $B^{n}$ is the closed unit ball in $R^{n}$, and $S^{n}$ is the one-point compactification of $R^{n} . S^{n}$ is triangulated so that $R^{n}$ and $B^{n}$ inherit their triangulations from $S^{n}$. When $m<n$, we identify $R^{m}$ with $R^{m} \times 0 \subset R^{n}$. Thus we have $R^{m} \subset R^{n} \subset S^{n}$ and $B^{m} \subset B^{n} \subset S^{n}$ for $m<n$. An $n$-cell (n-sphere, open $n$-cell) is a space homeomorphic to $B^{n}$ (resp. $S^{n}$, resp. $R^{n}$ ).

An $n$-manifold is a space $N$ such that each point of $N$ has a neighborhood whose closure is an $n$-cell; the interior of $N$ (denoted by Int $N$ ) is the set of points of $N$ which have open $n$-cell neighborhoods in $N$; the boundary of $N(\operatorname{denoted}$ by $\operatorname{Bd} N)$ is the complement of $N$-Int $N$ of Int $N$.

Let $M$ and $N$ be manifolds of dimension $m$ and $n$, respectively, with $M \subset$ Int $N$. $M$ is said to be locally flat in $N$ at the point $x \in \operatorname{Int} M$ if $x$ has a neighborhood $U$ in $N$ such that $(U, U \cap M) \approx\left(R^{n}, R^{m}\right)$; i.e., the pairs $(U, U \cap M)$ and $\left(R^{n}, R^{m}\right)$

Received by the editors April 5, 1966.

(1) This research constitutes a part of the author's doctoral dissertation under the direction of Professor J. C. Cantrell at the University of Georgia.

$\left({ }^{2}\right)$ The author is an NSF Graduate Fellow. 
are homeomorphic. $M$ is locally flat in $N$ at the point $x \in \mathrm{Bd} M$ if $x$ has a neighborhood $U$ in $N$ such that $(U, U \cap M) \approx\left(R^{n}, R^{m-1} \times[0, \infty)\right)$. $M$ is locally flat in $N$ if $M$ is locally flat at each point of $M$. In the special case when $M$ is an $m$-cell and $N$ is either $R^{n}$ or $S^{n}$, we say that $M$ is flat in $N$ if $(N, M) \approx\left(N, B^{m}\right)$.

In this paper, a polyhedron or complex will be understood to be finite unless otherwise stated. A combinatorial n-manifold is an $n$-manifold $N$ which has a locally finite triangulation in which the link of each vertex is either a combinatorial $(n-1)$-sphere or a combinatorial $(n-1)$-ball.

1. Embeddings which intersect the boundary. For the first two sections let the following be fixed: a (finite) $k$-complex $K$; a compact combinatorial $n$-manifold $N$; a closed subset $A$ of $K$; and an embedding $\phi$ of $A$ into $\mathrm{Bd} N$.

Let $\Phi$ be any set of embeddings $f: K \rightarrow N$ such that $f \mid A=\phi$ and $f \mid K-A$ is an embedding of $K-A$ into Int $N$. Let $d$ denote (ambiguously) a fixed metric for $N$ and the uniform metric on $\Phi$ induced by $d$ on $N$.

Definition. Let $X$ be a closed subset of $N, \varepsilon>0$. An $\varepsilon$-push $h$ of $(N, X ; \operatorname{Bd} N)$ is a homeomorphism of $N$ such that

(1) $h$ is an $\varepsilon$-homeomorphism of $N$ onto itself; i.e., $d(x, h(x))<\varepsilon$ for all $x$ in $N$;

(2) $h$ is the identity outside of the $\varepsilon$-neighborhood of $X$;

(3) $h$ is the identity on $\mathrm{Bd} N$; and

(4) $h$ is isotopic to the identity on $N$ through homeomorphisms satisfying (1), (2), and (3) above.

Definition. A subset $F$ of $\Phi$ is called solvable provided that for each $\varepsilon>0$ there is a $\delta=\delta(F, \varepsilon)>0$ such that if $f, g \in F$ and $d(f, g)<\delta$ then there is an $\varepsilon$-push $h$ of $(N, f(K) ; \operatorname{Bd} N)$ such that $h f=g$.

Notice that the above definition of solvability is a slight modification of that in [12]; however, the proofs of Theorems 4.3 and 4.4 of [12] need virtually no modification to yield the two lemmas below.

LEMMA 1.1. Let $F \subset \Phi$, and suppose that for each $f \in \Phi$ and $\varepsilon>0$ there is an $\varepsilon$-push $h$ of $(N, f(K) ; \mathrm{Bd} N)$ such that $h f \in F$. If $F$ is solvable then so is $\Phi$.

Lemma 1.2. Let $F_{1}$ and $F_{2}$ be subsets of $\Phi$, and suppose that each of $F_{1}$ and $F_{2}$ is dense in $\Phi$. If $F_{1}$ and $F_{2}$ are solvable then $F_{1} \cup F_{2}$ is solvable; in fact, given $\varepsilon>0$, one may take $\delta\left(F_{1} \cup F_{2}, \varepsilon\right)$ to be the minimum of $\left\{\delta\left(F_{1}, \varepsilon / 6\right), \delta\left(F_{2}, \varepsilon / 6\right)\right\}$.

The next definition and lemma are based on the approximation techniques used by Bryant in [5].

Definition. A subset $F$ of $\Phi$ is called weakly solvable provided that for each $\varepsilon>0$ there is a $\delta=\delta_{w}(F, \varepsilon)>0$ such that if $f, g \in F, d(f, g)<\delta$, and $f$ and $g$ agree on a neighborhood of $A$ in $K$, then there is an $\varepsilon$-push $h$ of $(N, f(K) ; \operatorname{Bd} N)$ such that $h f^{\prime}=g$. 
LEMMA 1.3. Let $F$ be any subset of $\Phi$, and for each $g \in F$ let $F_{g}=\{f \in F: f$ agrees with $g$ on a neighborhood of $A$ in $K\}$. If $F_{g}$ is dense in $F$ for each $g \in F$, and if $F$ is weakly solvable, then $F$ is solvable.

Proof. Let $\varepsilon>0$, and let $\delta=\delta_{w}(F, \varepsilon / 6)$ be given for $\varepsilon / 6$ by the weak solvability of $F$. Suppose that $f, g \in F$ and that $d(f, g)<\delta$. Note that $F_{f}$ and $F_{g}$ are solvable dense subsets of $F$, and in fact $\delta\left(F_{f}, \varepsilon^{\prime}\right)=\delta\left(F_{g}, \varepsilon^{\prime}\right)=\delta_{w}\left(F, \varepsilon^{\prime}\right)$ for each $\varepsilon^{\prime}>0$. Hence $F_{f} \cup F_{g}$ is solvable by Lemma 1.2 , and $\delta\left(F_{f} \cup F_{g}, \varepsilon\right)$ may be chosen to be $\min \left\{\delta\left(F_{f}, \varepsilon / 6\right), \delta\left(F_{g}, \varepsilon / 6\right)\right\}=\delta_{w}(F, \varepsilon / 6)=\delta$. Therefore, since both $f$ and $g$ belong to $F_{f} \cup F_{g}$ and $d(f, g)<\delta$, there is an $\varepsilon$-push $h$ of $(N, f(K)$; $\mathrm{Bd} N)$ such that $h f=g$. Hence $F$ is solvable.

2. Solvability of locally tame embeddings in the trivial range. The following definition of local tameness is the same as that used by Gluck in [12].

Definition. Let $X$ be a locally finite polyhedron topologically embedded in the manifold $M . X$ is said to be locally tame (with respect to the triangulation $f$ ) if there exist a locally finite complex $L$ and a homeomorphism $f: L \approx X$ which satisfy the following condition: given a point $x$ of $L$ there is a neighborhood $U$ of $f(x)$ in $M$ and a triangulation of $U$ as a combinatorial manifold with respect to which $\mid f^{-1}(U) f$ is piecewise linear.

An embedding $f$ of a locally finite complex $L$ into a manifold $M$ is called locally tame if $f(L)$ is locally tame in $M$.

Keeping $K, N, A$, and $\phi$ fixed as in $\S 1$, we make the following additional assumptions :

$\Phi=\{f: K \rightarrow N|f| A=\phi$, and $f \mid K-A$ is a locally tame embedding $K-A$

$F=\{f \in \Phi \mid f$ is piecewise linear on $K-A\}$, and for each $g \in F$,

into Int $N\}$.

$F_{g}=\{f \in F \mid f$ agrees with $g$ on a neighborhood of $A$ in $K\}$.

The remainder of this section is devoted to the proof of the following theorem.

THEOREM 2.1. If $n \geqq 2 k+2$ then $\Phi$ is solvable.

The proof is given in several steps, each of which has essentially been done in the literature. Assume $n \geqq 2 k+2$.

LEMmA 2.2. Given $\varepsilon>0$ and $f \in \Phi$, there is an $\varepsilon$-push $h$ of $(N, f(K) ; \mathrm{Bd} N)$ such that $h f \in F$.

Proof. The proof follows easily by applying Theorem 9.1 of [12] an infinite number of times.

LEMMA (BING-KISTER). Suppose that $K_{1}$ and $K_{2}$ are two closed locally finite $k$ complexes in $R^{n}$ and that $h$ is a piecewise linear homeomorphism of $K_{1}$ onto $K_{2}$ that 
does not move any point as far as $\varepsilon, n \geqq 2 k+2$. Suppose further that $L$ is a subcomplex of $K_{1}$ such that $\left(K_{1}-L\right)^{-}$is finite and $h \mid L=$ identity. Then there is an isotopy $h_{t}(0 \leqq t \leqq 1)$ of $R^{n}$ onto itself such that

(i) $h_{0}=$ identity,

(ii) $h_{1} \mid K_{1}=h$,

(iii) each $h_{t}$ is piecewise linear on $R^{n}$ and is the identity on $L$ and outside the $\varepsilon$-neighborhood of $K_{1}-L$,

(iv) each point of $R^{n}$ moves along a polygonal path of length less than $\varepsilon$.

The proof of the Bing-Kister lemma is essentially the same as the proof of Theorem 5.5 of [1].

Moreover, the proof of Lemma 2 of [13] can be used to prove the following lemma, substituting the Bing-Kister lemma for Proposition 1 of [13].

Lemma (Номма). For any $\varepsilon>0$ there is a $\delta=\delta(N, \varepsilon)>0$ such that if $\tilde{K}$ is a $k$-complex, $n \geqq 2 k+2$, and $f, g: \widetilde{K} \rightarrow N$ are embeddings which satisfy

(1) $f$ and $g$ agree on a neighborhood of $\tilde{A}=f^{-1}(\mathrm{Bd} N)$ in $\tilde{K}$,

(2) $f$ and $g$ are piecewise linear embeddings of $\tilde{K}-\tilde{A}$ into Int $N$, and

(3) $d(f, g)<\delta$,

then there is a piecewise linear $\varepsilon$-push $h$ of $(N, f(\widetilde{K}) ; \mathrm{Bd} N)$ such that $h f=g$; moreover, hf may be taken to agree with $f$ on some neighborhood of $\tilde{A}$ in $\tilde{K}$.

LEMMA 2.3. $F$ is weakly solvable.

Proof. This follows immediately from the Homma lemma above.

Lemma 2.4. For each $g \in F, F_{g}$ is dense in $F$.

Proof. Let $f, g \in F$; we will approximate $f$ by a member of $F_{g}$. To do this let $\varepsilon>0$ be given, and let $\delta=\delta(N, \varepsilon)$ be given by the Homma lemma.

Choose subcomplexes $L_{1}$ and $L_{2}$ of $K$ (taking subdivisions if necessary) which satisfy the following conditions (where $L_{i}^{\circ}$ and $L_{i}^{\circ}$ denote the interior and boundary of $L_{i}$ as subspaces of $K$ ).

(1) $A \subset L_{1}^{\circ} \subset L_{1} \subset L_{2}^{\circ}$,

(2) $d\left(f\left|L_{2}, g\right| L_{2}\right)<\delta$,

(3) $g\left(L_{1}\right) \cap f\left(\dot{L}_{2}\right)=\varnothing$.

Define embeddings $\bar{f}$ and $\bar{g}$ of $L_{1} \cup \dot{L}_{2}$ into $N$ by letting $\bar{f}\left|L_{1}=\bar{g}\left(L_{1}\right)=g\right| L_{1}$, $\bar{f}\left|\dot{L}_{2}=f\right| \dot{L}_{2}$, and $\bar{g}\left|\dot{L}_{2}=g\right| \dot{L}_{2}$. Thus $\bar{f}$ and $\bar{g}$ both agree with $g$ on $L_{1}$, but agree with $f$ and $g$ respectively on $\dot{L}_{2}$. Conditions (1), (2), and (3) above, together with the Homma lemma, show that there is a piecewise linear $\varepsilon$-push $h$ of

$$
\left(N, \bar{g}\left(L_{1} \cup \dot{L}_{2}\right) ; \operatorname{Bd} N\right)
$$

such that $h \bar{g}=\bar{f}$ and such that $h \bar{g}$ agrees with $g$ on a neighborhood of $A$ in $K$. 
Now define $f^{\prime}$ on $K$ by

$$
\begin{aligned}
f^{\prime} & =h g & & \text { on } L_{2}, \\
& =f & & \text { on } \overline{K-L_{2}} .
\end{aligned}
$$

$f^{\prime}$ is a mapping of $K$ into $N$ such that $f^{\prime}\left|L_{1}=g\right| L_{1}, f^{\prime} \mid L_{2}$ is an embedding, $f^{\prime} \mid K-A$ is piecewise linear, and $d\left(f^{\prime}, f\right)<\varepsilon+\delta \leqq 2 \varepsilon$. A general position argument completes the proof.

Proof of Theorem 2.1. Lemmas 1.3, 2.3, and 2.4 show that $F$ is solvable. But then Lemmas 1.1 and 2.2 show that $\Phi$ is solvable.

\section{An application.}

THEOREM 3.1. Let $N$ be a compact combinatorial n-manifold, $K$ a $k$-complex, $n \geqq 2 k+2$. Suppose that $f, g: K \rightarrow N$ are embeddings, $A=f^{-1}(\operatorname{Bd} N)$, and $f|A=g| A$. If $f \mid K-A$ and $g \mid K-A$ are locally tame embeddings of $K-A$ into Int $N$, and if $f$ and $g$ are homotopic through maps $f_{t}: K \rightarrow N(0 \leqq t \leqq 1)$ such that $f_{t}|A=f| A$ and $f_{t}(K-A) \subset N-f(A)$ for each $t$, then $f$ and $g$ are ambient isotopic leaving $\operatorname{Bd} N$ fixed.

Proof. First observe that we may assume that $f_{t}(K-A) \subset$ Int $N$ for each $t$. The reason for this is that there is a homotopy $f_{t}^{\prime}(0 \leqq t \leqq 1)$ of $N$ such that $f_{0}^{\prime}=f_{1}^{\prime}$ =identity, $f_{t}^{\prime} \mid f(A)=$ identity for each $t$, and $f_{t}^{\prime}(N-f(A)) \subset$ Int $N$ for $0<t<1$; $f_{t}^{\prime}(0 \leqq t \leqq 1)$ may be constructed by pushing $\mathrm{Bd} N-f(A)$ slightly into a collar for $\operatorname{Bd} N$. Then the homotopy $f_{t}^{\prime} f_{t}(0 \leqq t \leqq 1)$ has the desired properties.

Let $\Phi$ be the set of embeddings of $K$ into $N$ which agree with $\phi=f \mid A$ on $A$ and are locally tame embeddings of $K-A$ into Int $N$. By Theorem 2.1, $\Phi$ is solvable. (Note that $f$ and $g$ are in $\Phi$.) Let $\delta=\delta(\Phi, 1)>0$ be given by the solvability of $\Phi$ for $\varepsilon=1$. Choose a finite sequence $t_{0}=0<t_{1}<\cdots<t_{r}=1$ such that $d\left(f_{t_{i}}, f_{t_{1+1}}\right)<\delta / 3$ for $i=0, \ldots, r-1$. By a simplicial approximation and general position argument there are members $g_{1}, \ldots, g_{r-1}$ of $\Phi$ such that $d\left(g_{i}, f_{t_{i}}\right)<\delta / 3$ for $i=1, \ldots, r-1$. Letting $g_{0}=f$ and $g_{r}=g$, we have $d\left(g_{i}, g_{i+1}\right)<\delta$ for $i=0, \ldots, r-1$; hence there are 1-pushes $h_{i}$ of $\left(N, g_{i-1}(K) ; \operatorname{Bd} N\right)$ such that $h_{i} g_{i-1}=g_{i}, i=1, \ldots, r$. Define $h=h_{r} \circ \ldots \circ h_{1}$; then $h f=g, h \mid \mathrm{Bd} N=$ identity and $h$ is isotopic to the identity through homeomorphisms which are the identity on $\mathrm{Bd} N$. This completes the proof.

The following corollary is needed for the main result in $\$ 4$.

Corollary 3.2. Let $Q$ be an $n$-cell, $K$ a $k$-complex, $n \geqq 2 k+2$, and let $A$ be a closed subset of $K$. If $f, g: K \rightarrow Q$ are embeddings such that $f|A=g| A$ maps $A$ into $\operatorname{Bd} Q$ and such that $f \mid K-A$ and $g \mid K-A$ are locally tame embeddings of $K-A$ into Int $Q$, then $f$ and $g$ are ambient isotopic leaving $\mathrm{Bd} Q$ fixed.

Proof. It is clear that there is a homtopy $f_{t}(0 \leqq t \leqq 1)$ between $f$ and $g$ which satisfies the hypothesis of Theorem 3.1 , because $Q$ can be embedded in $R^{n}$ as a convex set. 
4. Taming a cell at its boundary. Before stating the main result of this paper, we will prove the following theorem.

TheOrem 4.1. Let $D$ be an $m$-cell in $S^{n}, n \geqq 4, m<n$, let $X$ be a closed set in $\mathrm{Bd} D$, and assume the following conditions:

(1) $D-X$ is locally flat in $S^{n}$;

(2) $X$ is cellular in $\mathrm{Bd} D$; and

(3) $X$ is cellular in $S^{n}$.

Then there is an embedding $\phi: B^{n} \rightarrow S^{n}$ such that $\phi\left(B^{m}\right)=D$ and $\phi\left(\operatorname{Bd} B^{n}\right)-X$ is locally flat in $S^{n}$.

Proof. (For the definition of cellularity and basic facts, see [3].) Since $X$ is cellular in $S^{n}$, there is a mapping $\pi$ of $S^{n}$ onto itself such that $X$ is the only (nondegenerate) inverse set of $\pi$; moreover, since $X$ is cellular in $\operatorname{Bd} D, \pi(D)$ is an $m$ cell. Since $\pi(D)$ is locally flat at each point other than $\pi(X) \in \mathrm{Bd} \pi(D)$, we may assume that $\pi(D)=B^{m}$ by Corollary 2.4 of [14]. Let $p=\pi(X)$, and let

$$
g=\pi^{-1} \mid S^{n}-\{p\} .
$$

The homeomorphism $g$ takes $S^{n}-\{p\}$ onto $S^{n}-X$, and $B^{m}-\{p\}$ onto $D-X$.

Let $k=n-m$, and let $j$ be the natural inclusion of $B^{m} \times R^{k}$ into $S^{n}$. Define $f$ on $(D-X) \times R^{k}$ by

$$
f(x, t)=g j\left(g^{-1}(x), t\right), x \in D-X, t \in R^{k} .
$$

$f$ is an embedding of $(D-X) \times R^{k}$ into $S^{n}-X$ which satisfies $f(x, 0)=x$ for $x \in D-X$, and $f\left((\operatorname{Bd} D-X) \times R^{k}\right)$ is locally flat in $S^{n}$. The $n$-cell $\phi\left(B^{n}\right)$ will be constructed in $f\left(\right.$ Int $\left.D \times R^{k}\right) \cup \mathrm{Bd} D$.

For each $x$ in Int $D$, let $\varepsilon(x)>0$ be chosen so that $f\left(x \times B_{x}\right)$ has diameter less than the distance from $x$ to $\mathrm{Bd} D$, where $B_{x}$ is the closed ball in $R^{k}$ with center 0 and radius $\varepsilon(x) . \varepsilon(x)$ may be chosen so that $\varepsilon$ is continuous on Int $D$ and so that $\varepsilon(x)=0, x \in \operatorname{Bd} D$ defines a continuous extension of $\varepsilon$ over all of $D$. Let

$$
N=\left\{(x, t) \in D \times R^{k}:\|t\| \leqq \varepsilon(x)\right\}
$$

and $N_{0}=$ (Int $\left.D \times R^{k}\right) \cap N . f \mid N_{0}$ can be extended to an embedding $F: N \rightarrow S^{n}$ by letting $F(x, t)=f(x, t)$ if $(x, t) \in N_{0}$ and $f(x, 0)=x$ if $x \in \mathrm{Bd} D$. It is clear from the construction of $N$ that $F$ is continuous and one-to-one.

The embedding $F$ takes $D \times 0$ onto $D$. Hence the proof of Theorem 4.1 will be complete as soon as we have shown that $(N, D \times 0) \approx\left(B^{n}, B^{m}\right)$ and that $F(\operatorname{Bd} N)-X$ is locally flat in $S^{n}$.

To prove the first of these assertions, let $\varepsilon_{1}$ be the continuous function which assigns to each point $x$ of $B^{m}$ the radius of the ball $B^{n} \cap H_{x}$, where $H_{x}$ is the $k$ plane in $R^{n}$ orthogonal to $R^{m}$ and passing through $x$. Then

$$
B^{n}=\left\{(x, t) \in B^{m} \times R^{k}:\|t\| \leqq \varepsilon_{1}(x)\right\} .
$$


If $h$ is a homeomorphism of $D$ onto $B^{m}, h$ can be extended to a homeomorphism $H: N \approx B^{n}$ by

$$
\begin{aligned}
H(x, t) & =\left(h(x), \varepsilon_{1}(x) t / \varepsilon(x)\right), & & x \in \operatorname{Int} D \\
& =h(x), & & x \in \operatorname{Bd} D .
\end{aligned}
$$

To see that $F(\mathrm{Bd} N)$ is locally flat at a point not in $\mathrm{Bd} D$, an argument similar to the one in the preceding paragraph will suffice. If $x$ is a point of $\operatorname{Bd} D-X$, then the homeomorphism $F^{-1}$ can be extended to a homeomorphism of a neighborhood of $x$ in $S^{n}$ in the following way: first extend over a neighborhood in $f\left((D-X) \times R^{k}\right)$ by $f^{-1}$, and then extend over a neighborhood in $S^{n}$ using the local flatness of $f\left((\mathrm{Bd} D-X) \times R^{k}\right)$. (Actually, there is no range for this last extension to map into. However, $D \times R^{k}$ may be thought of as being embedded in $R^{n}$ in such a way that $\mathrm{Bd} D \times R^{k}$ is locally flat.) Thus $F(\operatorname{Bd} N)-X$ is locally flat in $S^{n}$, and the theorem is established.

REMARK. The above theorem can be thought of in two ways. First, it gives a way to construct higher dimensional wild cells from lower dimensional ones; and second, it provides a method of taming lower-dimensional cells by knowing that a top-dimensional cell is tame. It is the second application which is used in the following theorem.

TheOREM 4.2. Let $D$ be an $m$-cell in $S^{n}$, let $X$ be a k-polyhedron in $\mathrm{Bd} D$, $n \geqq 2 k+4$, and assume that the following conditions hold:

(1) $D-X$ is locally flat in $S^{n}$,

(2) $X$ is locally tame in $S^{n}$, and

(3) $X$ is locally tame in $\mathrm{Bd} D$.

Then $D$ is flat in $S^{n}$.

Proof. The proof is divided into two cases. First, the theorem is proved assuming that $D$ is a top-dimensional cell. In the second case, Theorem 4.1 is used to "fatten up" a lower-dimensional cell into an $n$-cell.

Case 1. $m=n$. Thus $D$ is an $n$-cell in $S^{n}$ whose boundary is locally flat at each point except possibly at points in $X$. Let $D_{1}$ be an $n$-cell in Int $D$ such that $D$-Int $D_{1}$ is an $n$-annulus. Let $Q=\left(S^{n}-D\right)^{-}$and $Q_{1}=\left(S^{n}-D_{1}\right)^{-} \cdot Q_{1}$ is an $n$-cell by [3]. In order to show that $Q$ is an $n$-cell (which is equivalent to showing that $D$ is flat) we will construct mappings $\phi$ of $Q_{1}$ onto $Q$ and $\psi$ of $Q_{1}$ onto itself. $\phi$ and $\psi$ will have precisely the same nondegenerate inverse sets, so that the composition $\phi \psi^{-1}$ will be a homeomorphism of $Q_{1}$ onto $Q$.

Construction of $\phi$. Let $F$ be a homeomorphism of $D-$ Int $D_{1}$ onto $S^{n-1} \times[0,1]$ such that $F\left(\operatorname{Bd} D_{1}\right)=S^{n-1} \times 1$. It follows from Theorem 1 of [4] that $F$ can be extended to an embedding of $U \cup X$ into $S^{n-1} \times[-1,2]$, where $U$ is an open set in $S^{n}$ containing $D$-Int $D_{1}-X$. (We denote the extension by $F$.) Also, by Theorem 1.1 of [12] and assumption (3), we may assume that $F(X)$ is piecewise linearly 
embedded in $S^{n-1} \times 0$. Choose a complex $K$, linearly embedded in $S^{n-1}$, such that $K \times 0=F(X)$, and let $f=F^{-1} \mid K \times[0,1]$. Let $V$ be an open set in $S^{n-1} \times[-1,2]$ such that $\left(S^{n-1} \times[0,1]-F(X)\right) \subset V \subset F(U)$ and such that $\bar{V}-F(X) \subset F(U)-F(X)$. Clearly there is a mapping $\delta$ of $S^{n-1} \times[-1,2]$ onto itself such that $\delta=$ identity outside of $V$ and $\phi\left(S^{n-1} \times 1\right)=S^{n-1} \times 0$, and such that the nondegenerate inverse sets of $\phi$ are precisely the sets $x \times[0,1], x \in K$. Define $\phi$ on $Q_{1}$ by

$$
\begin{aligned}
\phi & =F^{-1} \tilde{\varphi} F \text { on } U \cap Q_{1} \\
& =\text { identity on } Q_{1}-U .
\end{aligned}
$$

$\phi$ is a mapping of $Q_{1}$ onto $Q$ whose nondegenerate inverse sets are precisely the sets $f(x \times[0,1]), x \in K$.

Construction of $\psi$. Let $G$ be a homeomorphism of $Q_{1}$ onto $I^{n}$. (Here $I^{1}=[0,1]$ and $I^{n}=I^{n-1} \times I^{1}$.) Again applying Theorem 1.1 of [12], we may assume that $G f \mid K \times 1$ is a piecewise linear embedding of $K \times 1$ into the interior of $I^{n-1} \times 1 \subset I^{n}$. The embedding $G f$ is clearly locally tame on $K \times(0,1)$, and $G f(K \times 0)$ is locally tame in $I^{n}$ by condition (2). Therefore $G f$ is a locally tame embedding of $K \times[0,1)$ into Int $I^{n}$ by Theorem 1 of [5]. Define $g: K \times[0,1] \rightarrow I^{n}$ by

$$
g(x, t)=(G f(x, 1),(t+1) / 2), x \in K, t \in[0,1] .
$$

$g$ is a locally tame embedding which agrees with $G f$ on $K \times 1$. Applying Corollary 3.2, there is a homeomorphism $H$ of $I^{n}$ onto itself such that $H G f=g$. But clearly there is a mapping $\tilde{\psi}$ of $I^{n}$ onto itself whose nondegenerate inverse sets are precisely the sets $g(x \times[0,1]), x \in K$, so define $\psi$ on $Q_{1}$ by $\psi=G^{-1} H^{-1} \tilde{\psi} H G$. $\psi$ is a mapping of $Q_{1}$ onto itself whose nondegenerate inverse sets are precisely the sets $f(x \times[0,1]), x \in K$.

Case 2. $m<n$. Suppose temporarily that $X$ is cellular in both $S^{n}$ and $\operatorname{Bd} D$. Then, by Theorem 4.1, there is an $n$-cell $\tilde{D}$ in $S^{n}$ such that $D \subset \tilde{D},(\tilde{D}, D) \approx\left(B^{n}, B^{m}\right)$, and Bd $\tilde{D}-X$ is locally flat in $S^{n}$. But $\tilde{D}$ is then flat by Case 1 , so that the homeomorphism $(\tilde{D}, D) \approx\left(B^{n}, B^{m}\right)$ can be extended to one of $S^{n}$ onto itself. (Clearly $X$ is locally tame in $\mathrm{Bd} \tilde{D}$ since $\mathrm{Bd} D$ is locally tame in Bd $\tilde{D}$.) Thus the theorem is established in the special case in which $X$ is cellular in both Bd $D$ and $S^{n}$.

Consider now the general case with no restrictions on $X$ other than local tameness. Let $x$ be a point of $X$. Since $X$ is locally tame in $\mathrm{Bd} D$, there is a neighborhood $V$ of $x$ in $D$ and a triangulation of $V$ as a combinatorial manifold which contains $V \cap X$ as a subcomplex and $x$ as a vertex. Let $\left(R, R_{0}\right)$ be the closed star of $x$ in the second barycentric subdivision of $(V, V \cap X)$. Then $R$ is an $m$-cell and $R$ is locally flat in $S^{n}$ except possibly at the points of $R \cap X=R_{0} \subset \mathrm{Bd} R$. Moreover, $R_{0}$ is tame and cellular in both $\mathrm{Bd} R$ and $S^{n}$. ( $R_{0}$ is cellular because it is a tame collapsible polyhedron.) It follows that $R$ is flat in $S^{n}$ and hence that $D$ is locally flat at $x$. Therefore $D$ is locally flat at every point and must be flat. 
CoRollary 4.3. Let $D$ be a cell in $S^{n}$ and $E$ a $k$-cell in $\mathrm{Bd} D, n \geqq 2 k+4$. If $D-E$ is locally flat in $S^{n}, E$ is locally flat in $S^{n}$, and $E$ is locally flat in $\operatorname{Bd} D$ then $D$ is flat in $S^{n}$.

Proof. Local flatness implies local tameness.

5. Applications. The first theorem in this section provides a method for taming cells whose "bad point set" is not polyhedral.

THEOREM 5.1. Let $D$ be a cell with locally flat interior in $S^{n}$, let $B$ denote the set of points of $\mathrm{Bd} D$ at which $D$ fails to be locally flat, and let $B_{0}$ be an open-closed subset of $B$. If $B_{0} \neq \varnothing$ then $B_{0}$ cannot be contained in $X \cap Y$, where $X$ is a tame $k$-polyhedron in $\mathrm{Bd} D$ and $Y$ is a tame l-polyhedron in $S^{n}, n \geqq 2 k+4, n \geqq 2 l+2$.

Proof. Suppose that such $X$ and $Y$ exist. Then, by Theorem 1 of [5], $X$ is tame in $S^{n}$.

Let $f: K \approx X$ be an embedding of the complex $K$ into $\mathrm{Bd} D$ such that $f$ is piecewise linear with respect to some triangulation of $D$ as a combinatorial ball. Since $f^{-1}\left(B_{0}\right)$ and $f^{-1}\left(B-B_{0}\right)$ are disjoint closed subsets of $K$, we may assume that $f(K) \cap\left(B-B_{0}\right)=\varnothing$.

Let $C(K)$ denote the cone over $K$, and extend $f$ to a piecewise linear embedding $F$ of $C(K)$ into $D$ which takes $C(K)-K$ into Int $D$. Let $N$ be a regular neighborhood of $F(C(K))$ which does not intersect $B-B_{0} . N$ is an $m$-cell in $D$, and $N$ is locally flat in $S^{n}$ except possibly at the points of $N \cap X=\mathrm{Bd} N \cap X=X$. Since $X$ is tame in $\operatorname{Bd} N$ and in $S^{n}, N$ is flat in $S^{n}$, and hence $D$ is locally flat at the points of $B_{0}$. This is a contradiction.

REMARK. (1) Let $D$ be a cell with locally flat interior in $S^{n}$, and let $B$ denote the set of points of $\mathrm{Bd} D$ at which $D$ fails to be locally flat. Corollary 2.5 of [14] shows that if $n \geqq 4$ and $B \neq \varnothing$ then $B$ is a perfect set and hence must contain a Cantor set. Theorem 5.1 above implies that if $n \geqq 6$ and $B$ is a Cantor set then $B$ must be wild in either $\mathrm{Bd} D$ or $S^{n}$.

(2) The examples of wild cells in [2] can be used to show that the condition that $X$ be locally tame in $S^{n}$ is necessary in Theorem 4.2. The author does not know whether the condition that $X$ be locally tame in Bd $D$ is necessary.

We conclude this section by interpreting Theorems 4.2 and 4.3 for embeddings of manifolds. Theorem 5.1 has a similar generalization.

THEOREM 5.2. Let $M$ and $N$ be combinatorial manifolds of dimension $m$ and $n$, respectively, with $M \subset$ Int $N$. Suppose that $M-X$ is locally flat in $N$, where $X$ is a $k$-polyhedron in $\mathrm{Bd} M, n \geqq 2 k+4$. If $X$ is locally tame in both $\mathrm{Bd} M$ and $N$ then $M$ is locally flat in $N$.

Theorem 5.2 is proved in a manner similar to the proof of 5.3 below. 
THEOREM 5.3. Let $K, M$, and $N$ be (topological) manifolds of dimension $k, m$, and $n$, respectively, with $K \subset \mathrm{Bd} M \subset M \subset$ Int $N$ and $n \geqq 2 k+4$. If $M-K$ and $K$ are locally flat in $N$ and $K$ is locally flat in $\mathrm{Bd} M$ then $M$ is locally flat in $N$.

Proof. Let $x \in K$. Since $K$ is locally flat in $\operatorname{Bd} M$, there is an $m$-cell $D$ in $M$ such that $D \cap \mathrm{Bd} M$ is an $(m-1)$-cell containing $x$ as an interior point, $D \cap K$ is a $k$-cell locally flat in $\mathrm{Bd} D$, and $D-K$ is locally flat in $N . D$ may be chosen small enough so that $D$ lies in an open $n$-cell $U$ in $N$. It follows from Corollary 4.3 that $D$ is locally flat in $U$, and hence that $M$ is locally flat in $N$ at the point $x$. Thus $M$ is locally flat in $N$.

6. Taming a cell at interior points. Let $\beta(n, m, m-1)$ denote the following conjecture.

Conjecture $\beta(n, m, m-1)$. Let $D_{1}$ and $D_{2}$ be two flat $m$-cells in $S^{n}$ such that $D_{1} \cap D_{2}=\operatorname{Bd} D_{1} \cap \mathrm{Bd} D_{2}$ is an $(m-1)$-cell which is locally flat in both $\mathrm{Bd} D_{1}$ and $\mathrm{Bd} D_{2}$. Then $D_{1} \cup D_{2}$ is a flat cell.

$(\beta(n, m, m-1)$ is one in the class of conjectures considered by Cantrell in [8].)

In [10], Černavskii anounces that $\beta(n, m, m-1)$ is true whenever $n \geqq 5$ and $m \neq n-2$. This section extends the results in $\S 4$ to the interior of a cell in any dimension for which $\beta(n, m, m-1)$ holds.

Definition. Let $X$ be a locally finite polyhedron topologically embedded in the $n$-manifold $N ; l$ is a nonnegative integer. $X$ is said to be locally l-tame in $N$ if there exist a locally finite complex $K$ and a homeomorphism $f: K \approx X$ such that the following holds: given a point $x$ of $K$, there is a neighborhood $U$ of $f(x)$ in $N$ and a homeomorphism $h: \bar{U} \approx B^{n}$ such that $h f \mid f^{-1}(\bar{U})$ is a piecewise linear embedding of $f^{-1}(\bar{U})$ into $B^{n-l}$.

This definition seems rather complicated, and a discussion of the relations between different degrees of local tameness and local embeddability is beyond the scope of this paper. However, it is often easy to decide whether or not a particular embedding is locally $l$-tame for some $l \geqq 1$, and for this reason we use the definition without further discussion. (It is easy but interesting to list the relations between local 0-tameness, local 1-tameness, local 2-tameness, and local flatness in the case of a 2-manifold in a 4-manifold.)

THEOREM 6.1. Let $D$ be an $m$-cell in $S^{n}$, let $X$ be a $k$-polyhedron in $D, n \geqq 2 k+4$, and assume that the following hold:

(1) $D-X$ is locally flat in $S^{n}$;

(2) $X$ is locally tame in $S^{n}$; and

(3) $X$ is locally 1-tame in $D$.

If $\beta(n, m, m-1)$ is true then $D$ is flat in $S^{n}$. 
Proof. Let $x$ be a point of $X$ which lies in Int $D$. Since $X$ is locally 1-tame in $D$, there is a neighborhood $U$ of $x$ in $D$ and a homeomorphism $h: \bar{U} \approx B^{m}$ such that $h(\bar{U} \cap X)$ is a subcomplex of $B^{m-1}$. Clearly we may assume that $\mathrm{Bd} \bar{U}$ is locally flat in Int $D$. Let $B_{+}\left(B_{-}\right)$be the set of points of $B^{m}$ whose last coordinates are nonnegative (resp. nonpositive). Then $B_{+} \cup B_{-}=B^{m}$ and

$$
B_{+} \cap B_{-}=\mathrm{Bd} B_{+} \cap \mathrm{Bd} B_{-}=B^{m-1} .
$$

Define $D_{1}=h^{-1}\left(B_{+}\right)$and $D_{2}=h^{-1}\left(B_{-}\right)$. Clearly $D_{1}$ and $D_{2}$ are locally flat in $S^{n}$ except possibly at the points of $\bar{U} \cap X$. But $\bar{U} \cap X$ is a $k$-polyhedron which is locally tame in each of $\mathrm{Bd} D_{1}, \operatorname{Bd} D_{2}$, and $S^{n}$. Hence, since $n \geqq 2 k+4, D_{1}$ and $D_{2}$ are flat in $S^{n}$ by Theorem 4.2. Finally, by the assumption that $\beta(n, m, m-1)$ is true, $D_{1} \cup D_{2}=\bar{U}$ is a flat cell in $S^{n}$, and $D$ is locally flat at the point $x$. Thus Int $D$ is locally flat in $S^{n}$.

Now let $x$ be a point of $X$ in $\mathrm{Bd} D$. Since $X$ is locally tame in $D$, there is a neighborhood $V$ of $x$ in $D$ such that $\bar{V}$ is an $m$-cell and $X \cap \mathrm{Bd} \bar{V}$ is a locally tame polyhedron in Bd $\bar{V}$ and in $S^{n}$. We choose $V$ so that $\bar{V}-X$ is locally flat in $S^{n}$. But then $\bar{V}-(X \cap \mathrm{Bd} \bar{V})$ is locally flat since Int $D$ is locally flat, and $\bar{V}$ is flat in $S^{n}$ by Theorem 4.2. Thus $D$ is locally flat at $x$, and $D$ is a flat cell in $S^{n}$.

Corollary 6.2. Let $D$ and $E$ be cells in $S^{n}, E \subset D$, such that $(D, E) \approx\left(B^{m}, B^{k}\right)$, $n \geqq 2 k+4$. If $D-E$ and $E$ are locally flat in $S^{n}$ (and if $\beta(n, m, m-1)$ is true) then $D$ is flat in $S^{n}$.

REMARK. (1) $\beta(n, n-2, n-3)$ is known to be false for $n \geqq 3$. Moreover, the conclusion of Corollary 6.2 is false when $m=n-2$. See [8] and Corollary 2.6 of [14].

(2) The theorems of $\S 6$ can be generalized in the same way that $\S 5$ generalizes $\S 4$.

\section{REFERENCES}

1. R. H. Bing and J. M. Kister, Taming complexes in hyperplanes, Duke Math. J. 31 (1964), 491-511.

2. W. A. Blankinship, Generalization of a construction of Antoine, Ann. of Math. 53 (1951), 276-297.

3. M. Brown, A proof of the generalized Schoenflies theorem, Bull. Amer. Math. Soc. 66 (1960), 74-76.

4. - Locally flat embeddings of topological manifolds, Ann. of Math. 75 (1962), 331-341.

5. J. L. Bryant, Taming polyhedra in the trivial range, (to appear).

6. J. C. Cantrell, Almost locally flat embeddings of $S^{n-1}$ in $S^{n}$, Bull. Amer. Math. Soc. 69 (1963), 716-718.

7. - $n$-frames in euclidian $k$-space, Proc. Amer. Math. Soc. 15 (1964), 574-578.

8. - Some results concerning the union of flat cells, Duke Math. J. 32 (1965), 673-678.

9. J. C. Cantrell and C. H. Edwards, Jr., Almost locally flat imbeddings of manifolds, Michigan Math. J. 12 (1965), 217-223.

10. A. V. Cernavskiǐ, Isotopies in euclidean spaces, Russian Math. Surveys 19 (1964), 63-65. 
11. C. H. Edwards, Jr., Taming 2-complexes in high-dimensional manifolds, Duke Math. J. 32 (1965), 479-494.

12. H. Gluck, Embeddings in the trivial range, Ann. of Math. 81 (1965), 195-210.

13. T. Homma, On the imbeddings of polyhedra in manifolds, Yokohama Math. J. 10 (1962), 5-10.

14. C. Lacher, Locally flat strings and half-strings, Proc. Amer. Math. Soc. (to appear).

\section{UNIVERSITY OF GEORGIA,} Athens, Georgia 